PROFESIONALES Y HERRAMIENTAS PARA EL DESARROLLO LOCAL Y SUS SINERGIAS TERRITORIALES. EVALUACIÓN Y PROPUESTAS DE FUTURO IX Coloquio Nacional de Desarrollo Local del GTDL-AGE 

ANTONIO MARTÍNEZ PUCHE, XAVIER AMAT MONTESINOS, ISABEL SANCHO CARBONELL y DANIEL SANCHIZ CASTAÑO (EDS.)

\section{PROFESIONALES Y HERRAMIENTAS PARA EL DESARROLLO LOCAL Y SUS SINERGIAS TERRITORIALES. EVALUACIÓN Y PROPUESTAS DE FUTURO}

IX Coloquio Nacional de Desarrollo Local del GTDL-AGE

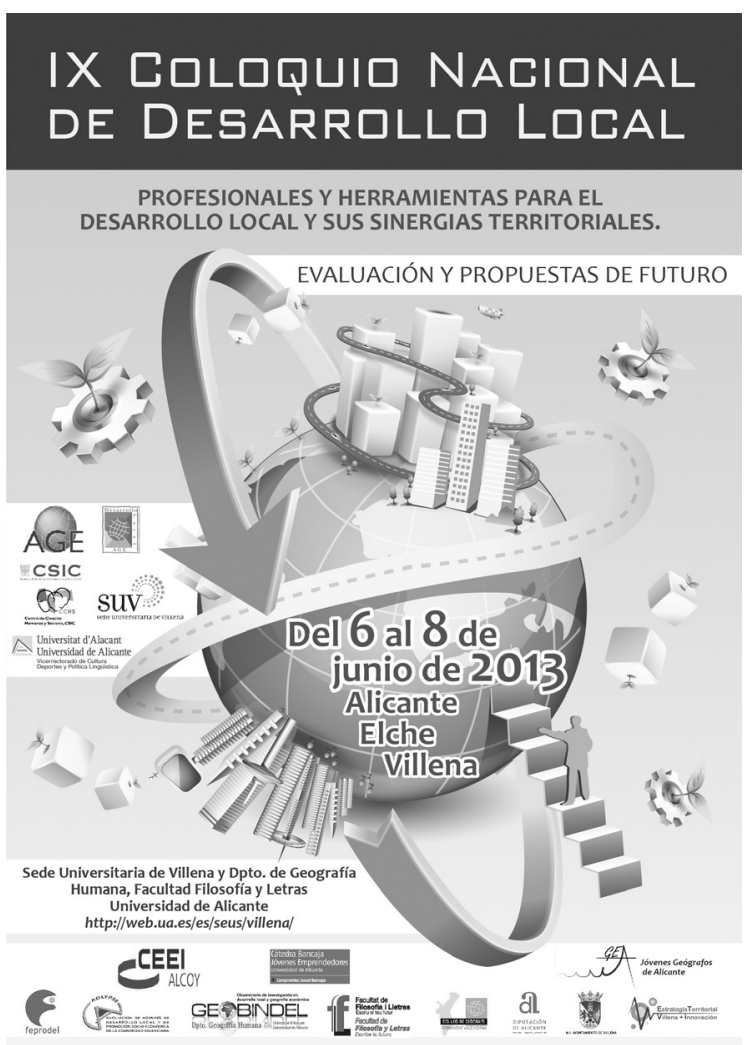


Este libro ha sido examinado y valorado por evaluadores ajenos a la Universidad de Alicante, con el fin de garantizar la calidad científica del mismo.

Publicacions de la Universitat d'Alacant

03690 Sant Vicent del Raspeig

Publicaciones@ua.es

http://publicaciones.ua.es

Telèfon: 965903480

(C) Antonio Martínez Puche, Xavier Amat Montesinos,

Isabel Sancho Carbonell y Daniel Sanchiz Castaño (eds.), 2016

(C) d'aquesta edició: Universitat d'Alacant

ISBN: 978-84-16724-00-0

Dipòsit legal: A 92-2016

Disseny de coberta: candela ink

Composició: Página Maestra (Miguel Ángel Sánchez Hernández)

Impressió i enquadernació: Guada Impresores

\section{unte \\ Unión de Editoriales
Universitarias Españolas \\ WWW.une.es
WWA}

Esta editorial es miembro de la UNE, cosa que garantiza la difusión y comercialización nacional y internacional de sus publicaciones.

Reservados todos los derechos. Cualquier forma de reproducción, distribución, comunicación pública o transformación de esta obra sólo puede ser realizada con la autorización de sus titulares, salvo excepción prevista por la ley. Diríjase a CEDRO (Centro Español de Derechos Repográficos, www.cedro.org) si necesita fotocopias o escanear algún fragmento de esta obra. 


\title{
LA MÚSICA COMO ELEMENTO DE PARTICIPACIÓN CIUDADANA Y DESARROLLO LOCAL
}

\author{
Loretta González de Bustos \\ Técnico en gestión cultural. Especialista en Gestión Cultural de Sociedades Musicales y Más- \\ ter en Desarrollo Local e Innovación Territorial \\ loretta.gonzalez@ua.es
}

\section{RESUMEN}

Esta comunicación trata de mostrar la importancia de la cultura valenciana musical, representada en su principal formación que son las bandas de música, las cuales conforman un patrimonio cultural que se caracteriza por un fuerte enraizamiento con el territorio, creador de identidad cultural local y dotadas de un extraordinario movimiento profesional y amateur representado por los músicos. Se trata de un bien patrimonial (en trámite como Bien de Interés Cultural por el Consell Valenciano) y una fuente de desarrollo local.

La cultura musical, representada por las bandas, incide en procesos de desarrollo local y de desarrollo económico debido a la importancia de sus manifestaciones: conciertos, festivales, concursos, escuelas de música, producción, venta y reparación de instrumentos musicales y toda una economía que gira en torno a esta manifestación artística. Son un recurso endógeno del desarrollo local que dota de una singular identidad a los valencianos y marca parte de su seña territorial. Es por ello que se hace necesaria una reflexión para dotar a la idea de cultura musical de una mayor importancia socioeconómica.

El patrimonio musical valenciano es una importante industria cultural porque la creación, producción, distribución y consumo de cultura genera riqueza. Sus estadísticas así lo avalan: 534 sociedades musicales para todo el territorio de la Comunidad Valenciana, 200.000 socios, 2.575 empleos. La territorialización cubre toda la geografía de los municipios, comarcas y provincias de la comunidad autónoma. Los resultados más visibles de esta industria cultural son los conciertos y la formación musical recibida por los músicos e impartida en las sociedades musicales. Estas sociedades musicales han tenido un continuado 
apoyo público, aunque en estos momentos han visto recortadas sus subvenciones fuertemente.

La innovación social necesaria para estos procesos de desarrollo local se manifestó en este fuerte fenómeno cultural desde un principio. La participación ciudadana se concretó en unión del trabajo de muchísimos ciudadanos vinculados con la música: desde el socio de la asociación musical de la que nacen la gran mayoría de estas bandas, el propio músico, el maestro, el gestor cultural y el gestor político. Estos han construido a las señas de identidad de este movimiento musical, propiciado por el innato talento local para hacer música, para hacer cultura. Igualmente, el voluntariado en cada uno de los sectores relacionados con el fenómeno musical permite que exista este fenómeno de bandas tan singular, también la amplia formación musical gestada en las Sociedades Musicales, el virtuosismo musical de los músicos y el saber hacer música de generación tras generación (know how) es lo que ha conducido a un apoyo por parte de las autoridades municipales y autonómicas para permitir la continuidad de esta industria cultural. Se trata, pues, de un patrimonio activo, un recurso tangible, un fuerte agente dinamizador sociocultural igualmente, mezcla de muchos empleos directos, indirectos e inducidos, y de una gran entrega en el trabajo voluntario de músicos socios afiliados.

Por ello, nuestra reflexión lleva de la mano una idea de estrategia de puesta en valor del patrimonio musical a través de una serie de cambios que conduzcan a su mantenimiento en el tiempo, a su sostenibilidad.

Palabras clave: cultura valenciana musical, cohesión social.

\section{THE MUSIC LIKE ELEMENT OF CIVIC PARTICIPATION AND LOCAL DEVELOPMENT}

\section{ABSTRACT}

The musical culture, represented by the bands, impacts in processes of local development and of economic development due to the importance of their manifestations: concerts, festivals, competitions, music schools, production, sale and repair of musical instruments and an entire economy that it rotates around this artistic manifestation. They are an endogenous resource of the local development that endows from a singular identity to the Valencian ones and it marks it leaves of their territorial sign. It is hence that it becomes necessary a reflection to endow to the idea of musical culture of a socioeconomic bigger importance. The musical Valencian patrimony is a cultural important industry because the creation, production, distribution and culture consumption generates wealth. Their statistics endorse this way it: 534 musical societies for the whole territory of the Valencian Community, 200.000 partners, 2.575 employments. The territorialización covers the whole geography of the municipalities, districts 
and counties of the autonomous community. The most visible results in this cultural industry are the concerts and the musical formation received by the musicians and imparted in the musical societies. These musical societies have had a continuous support public, although in these moments their grants have been clipped strongly.

Keywords: Valencian musical culture, social cohesion.

\section{INTRODUCCIÓN}

Esta comunicación trata de mostrar la importancia de la cultura valenciana musical, representada en su principal formación que son las bandas de música y cómo éstas conforman un patrimonio cultural que se caracteriza por un fuerte enraizamiento con el territorio, creador de identidad cultural local y dotadas de un extraordinario movimiento profesional y amateur representado por los músicos. Este es un bien patrimonial, pues las bandas de música están declaradas Bien de Interés Local por el Consell Valenciano en julio del año 2011.

Esta cultura musical incide en procesos de desarrollo local y de desarrollo económico, debido a la importancia de sus manifestaciones: conciertos, festivales, concursos, escuelas de música, producción, venta y reparación de instrumentos musicales y toda una economía que gira en torno a esta manifestación artística. Son un recurso endógeno de desarrollo local, y no simplemente un recurso inadvertido para el territorio; sin embargo, la música de banda dota de una singular identidad a los valencianos, marca parte de su seña territorial. Es por ello que se hace necesaria una reflexión para dotar a la idea de cultura musical de una mayor importancia socioeconómica.

El patrimonio musical valenciano es una importante industria cultural, que se caracteriza por un fuerte sentido colectivo de su identidad territorial, donde los músicos se dedican a tocar en bandas y a enseñar en escuelas musicales desde un enfoque amateur y profesional Sus estadísticas lo avalan: 534 sociedades musicales para todo el territorio de la Comunidad Valenciana, 200.000 socios y 2.575 empleos. La territorialización cubre toda la geografía de los municipios, comarcas y provincias de la comunidad autónoma. Los resultados más visibles de esta industria cultural son los conciertos y la formación musical recibida por los músicos e impartida en las sociedades musicales. Estas sociedades musicales han tenido un continuado apoyo público, aunque en estos momentos han visto recortadas sus subvenciones fuertemente.

La innovación social necesaria para estos procesos de desarrollo local se manifestó en este fuerte fenómeno cultural desde un principio. La participación ciudadana se concretó en la unión del trabajo de muchísimos ciudadanos vinculados con la música: desde el socio de la asociación musical de las que nacen la gran mayoría de estas bandas, el propio músico, el maestro, el gestor 
cultural y el gestor político han construido cada una de las señas de identidad de este movimiento musical. También es innovación social la amplia formación musical gestada en las Sociedades Musicales, el talento local de los músicos, el saber hacer música de generación tras generación y que ha conducido a un apoyo por parte de las autoridades municipales y autonómicas para permitir la continuidad de esta industria cultural. La música de banda es un patrimonio activo, un recurso tangible, son un fuerte agente dinamizador sociocultural igualmente, mezcla de muchos empleos directos, indirectos, inducidos y de una gran entrega en el trabajo voluntario de músicos socios afiliados.

Por ello, la reflexión lleva de la mano una idea de estrategia de puesta en valor del patrimonio musical a través de una serie de cambios que conduzcan a su mantenimiento en el tiempo, a su sostenibilidad.

\section{LAS SOCIEDADES MUSICALES Y EL TERRITORIO}

La existencia de las sociedades musicales es uno de los rasgos característicos más peculiares y diferenciadores de la comunidad autónoma. El fenómeno de las bandas musicales es un fenómeno reciente, cada vez más imbricado en las estructuras urbanas y territorializado de una forma sorprendentemente homogénea.

En términos cuantitativos, queda claro que la Comunidad Valenciana posee el número de Sociedades Musicales más elevado y nutrido de toda España. Los datos de este mismo año recogen la existencia de 529 sociedades en nuestra comunidad, repartidas como se ve en la tabla de datos que viene a continuación. Cada una de las sociedades tiene su propia banda musical, y entre todas ellas comprenden a unos 200.000 socios. Es de destacar que el número de músicos, ya federados o en formación (músicos potenciales), alcanza los 100.000, de tal modo que su continuidad y crecimiento parecen asegurados durante generaciones venideras. Estas cifras resultan especialmente significativas e importantes si tenemos en cuenta que nuestra comunidad tiene una población total de 5.111.706 habitantes (según el Instituto Valenciano de Estadística). Si restamos a ello la población extranjera (893.000 habitantes), que por lo general no participa en este tipo de sociedades y sus actividades, el resultado es que aproximadamente un $6 \%$ de la población de la comunidad está vinculada a las sociedades musicales.

En primer lugar, podemos decir que el reparto de estas 534 sociedades sigue, en líneas generales, criterios demográficos y de tradición. Valencia es la provincia más poblada y también la de mayor tradición musical, y por tanto aglutina más de la mitad de esas sociedades (bastantes de las más antiguas y más prestigiosas, como la de Llíria, surgen allí). Le siguen, según esa misma regla, Alicante y Castellón. Y la misma escala se aplica a la cantidad de socios y a la de alumnos. El siguiente gráfico nos sirve como comparativa: 


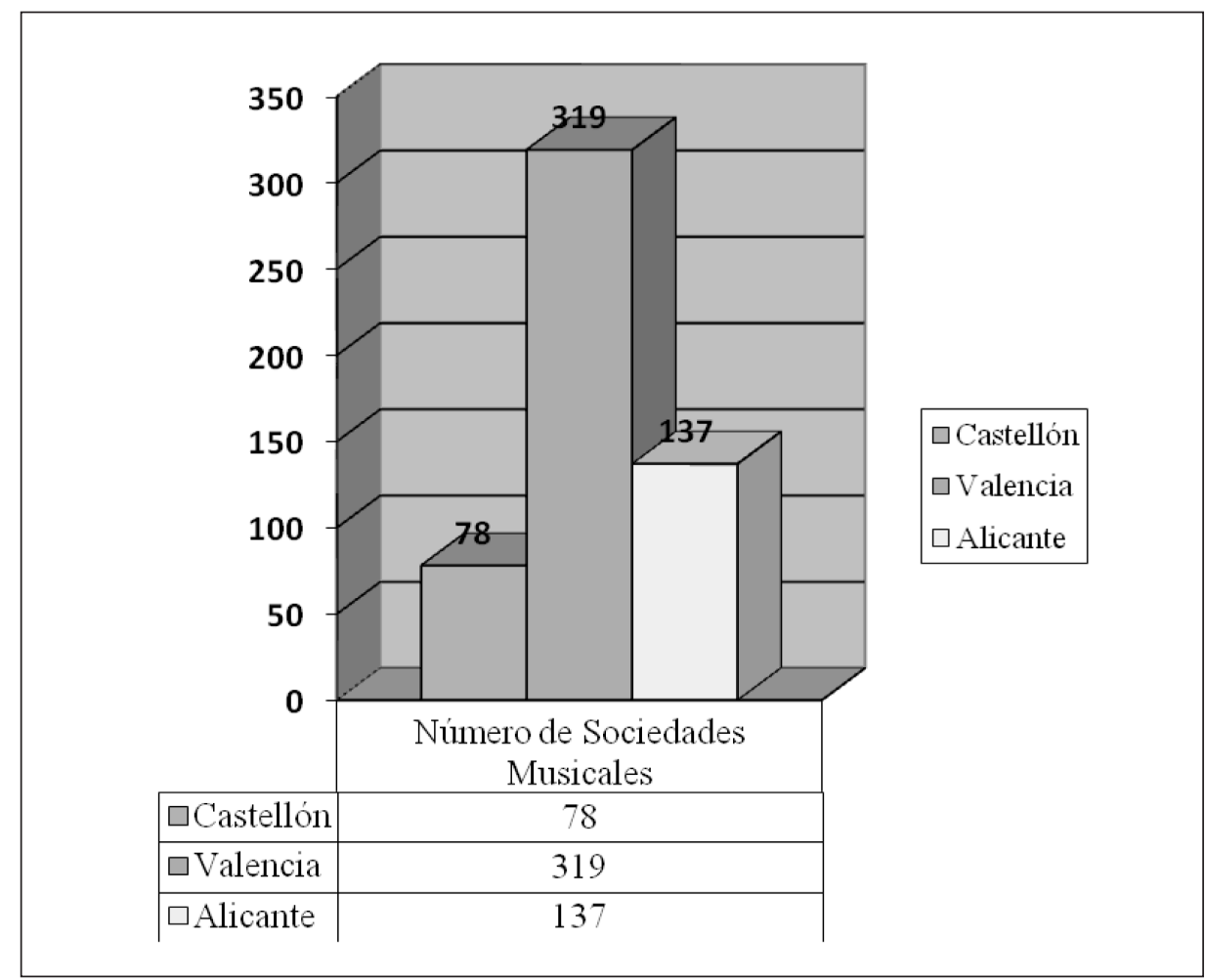

Figura 1. Sociedades musicales en la Comunidad Valenciana.

Fuente: Federación de Sociedades Musicales de la Comunidad Valenciana. Datos de 2011. Elaboración propia.

Esta distribución no implica un criterio de valor respecto a las formaciones mismas, sino que se atiene, como hemos dicho, a una cuestión puramente demográfica y de cultura musical, aunque es cierto que influyen también otros factores tales como la muy escasa movilidad de los músicos en este tipo de actividad. Como puede observarse en el gráfico siguiente, el crecimiento del número de bandas (y, por tanto, de sociedades musicales) en la Comunidad Valenciana durante los últimos catorce años no muestra que una provincia destaque sobremanera respecto a las otras. La diferencia porcentual entre la de mayor y la de menor crecimiento no alcanza los cinco puntos. Quiere esto decir que el crecimiento a este respecto muestra porcentajes similares en las tres provincias. Si acaso, son las provincias con menor número de sociedades musicales quienes tienen un mayor margen de crecimiento, y así se demuestra en la siguiente comparación de datos: 


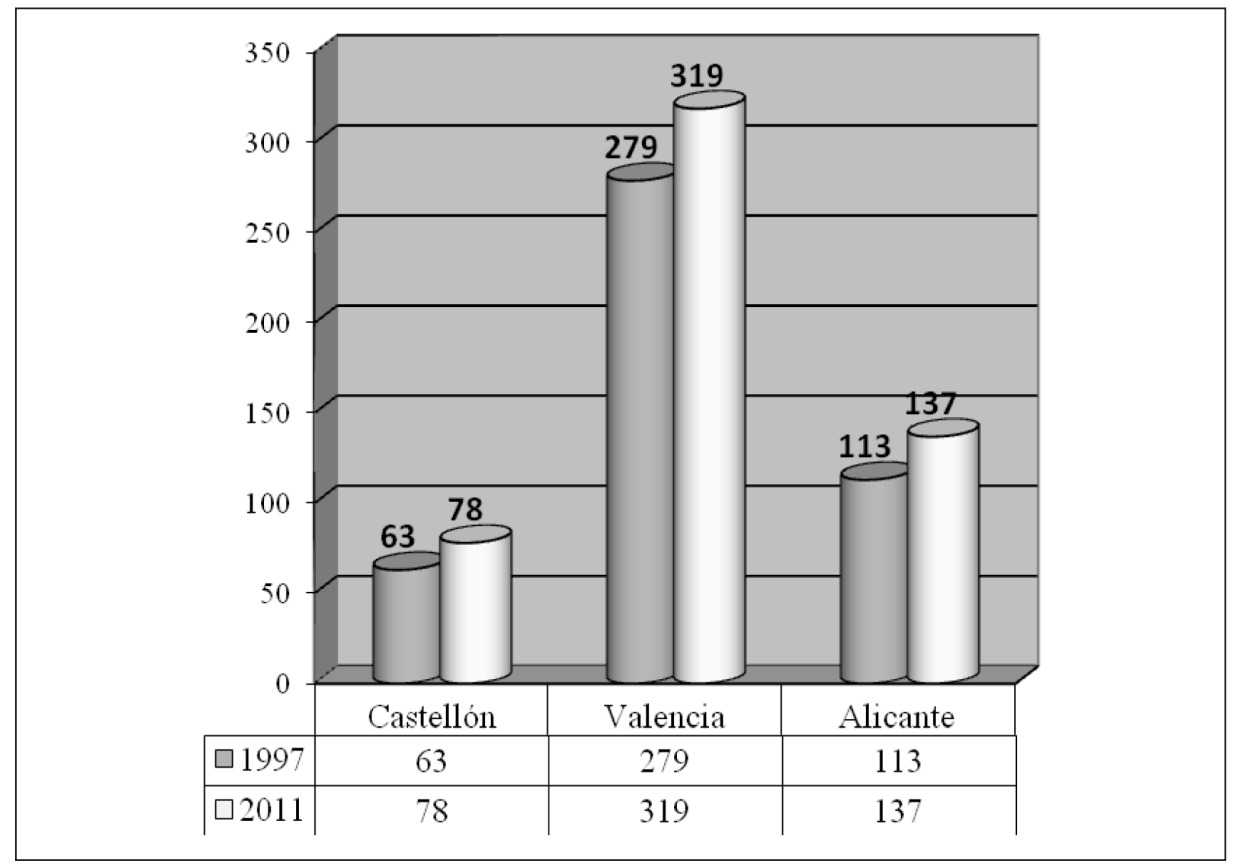

Figura 2. Comparativa del crecimiento de bandas por provincias.

Fuente: Federación de Sociedades Musicales de la Comunidad Valenciana.

Datos 1997 y 2011. Elaboración propia.

Cuadro 1. Crecimiento de las bandas de música

\begin{tabular}{|c|c|c|}
\hline PROVINCIA & BANDAS NUEVAS & \% CRECIMIENTO \\
\hline CASTELLÓN & 14 & $16.88 \%$ \\
\hline VALENCIA & 37 & $11.7 \%$ \\
\hline ALICANTE & 27 & $16.91 \%$ \\
\hline
\end{tabular}

Fuente: Federación de Sociedades Musicales de la Comunidad Valenciana. Datos 1997 y 2011. Elaboración propia.

A la luz de estos datos, la provincia de Valencia ha crecido un $11,70 \%$ sobre la cantidad anterior de sociedades musicales, a pesar de que el número de sociedades nuevas es mayor en esa provincia (37). Por su parte, Alicante creció en un 16'91\% y Castellón en un 16'88\%. Estos porcentajes sugieren dos cosas: en primer lugar, que la crisis económica de los últimos años ha afectado a todas las provincias, pues el porcentaje de crecimiento no es elevado para un período de catorce años. El mismo informe de la Universidad de Valencia señala que el 


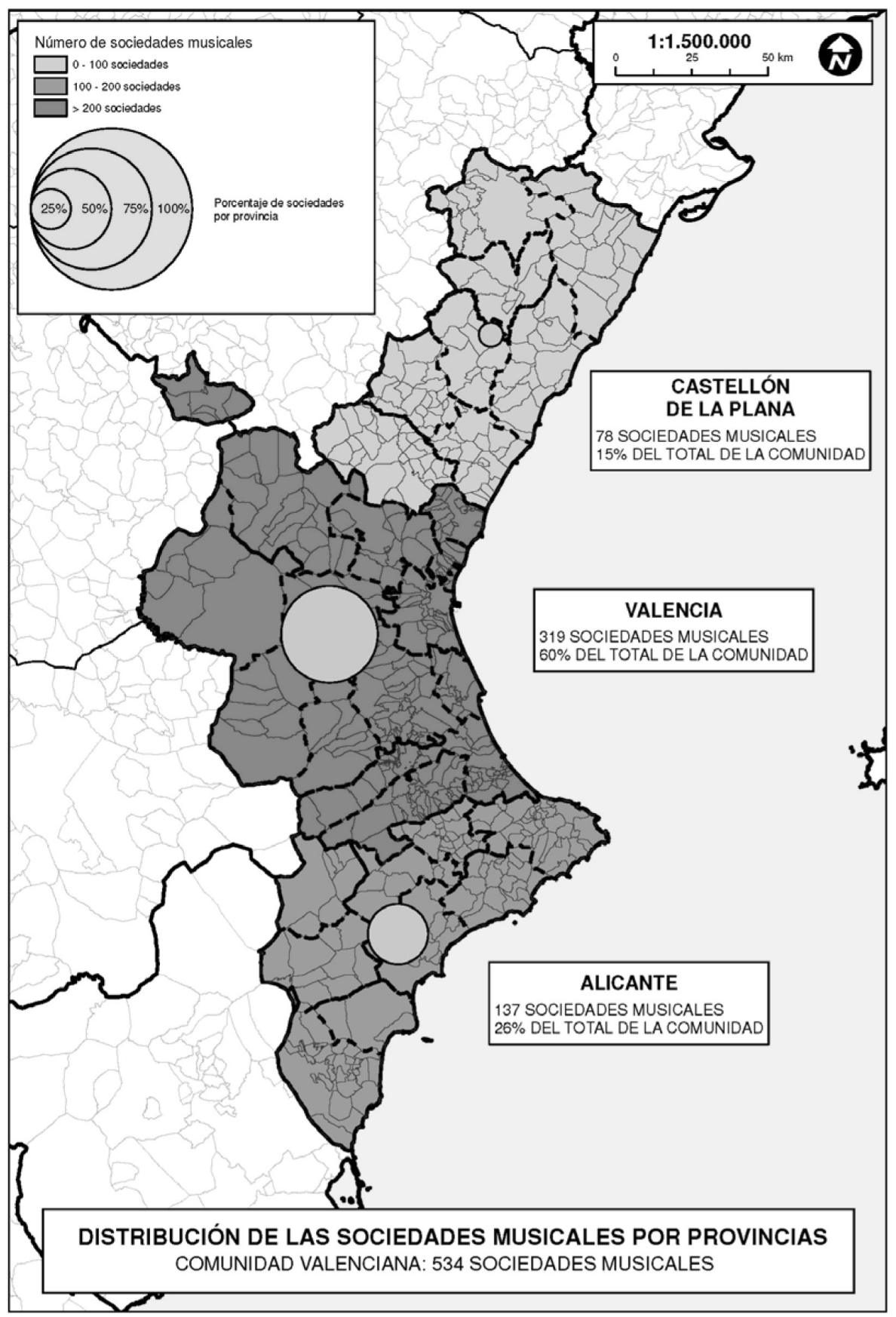

Figura 3. Número de sociedades musicales.

Fuente: elaboración propia. 


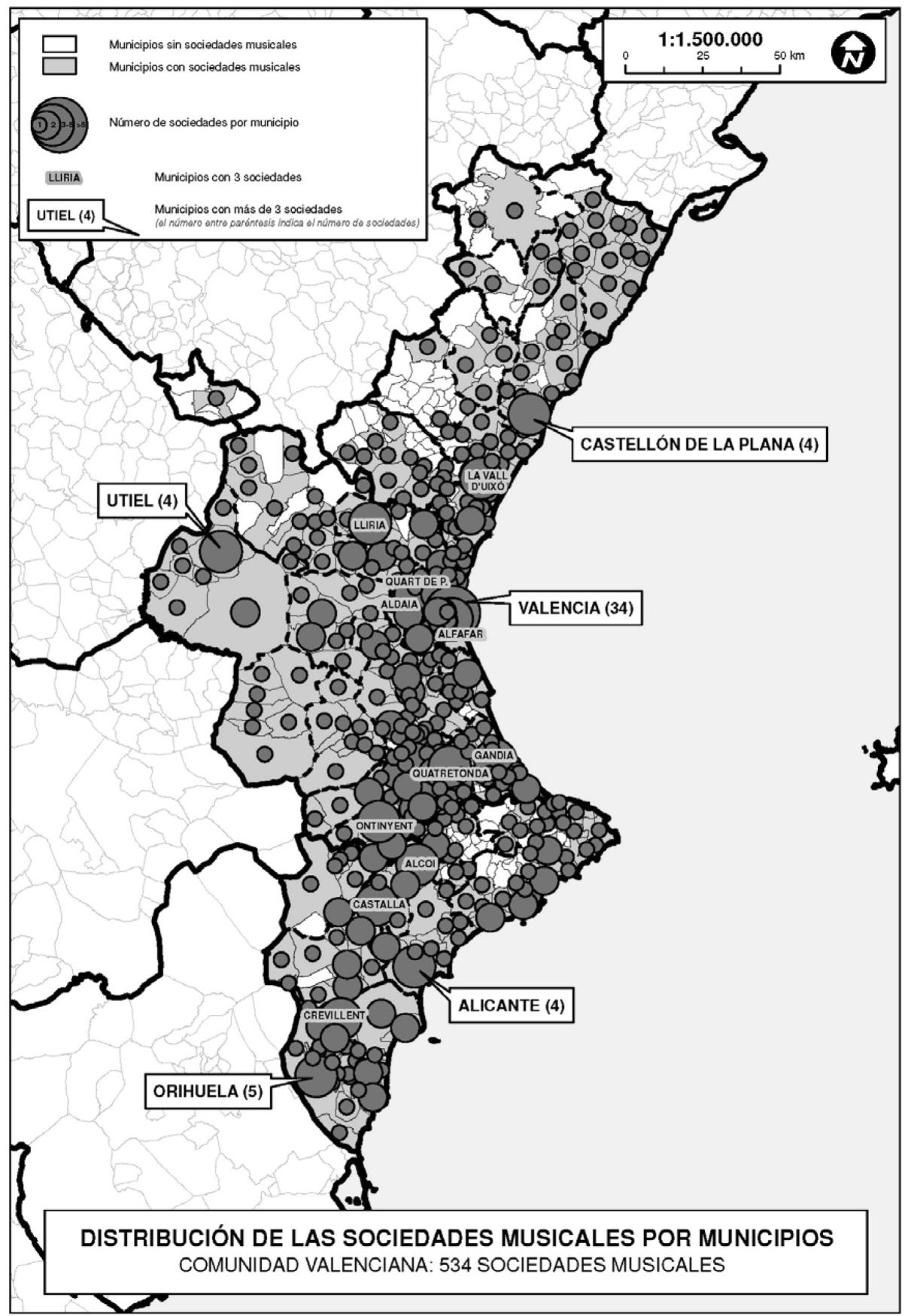

Figura 4. Distribución municipios con sociedades municipales.

Fuente: elaboración propia. 


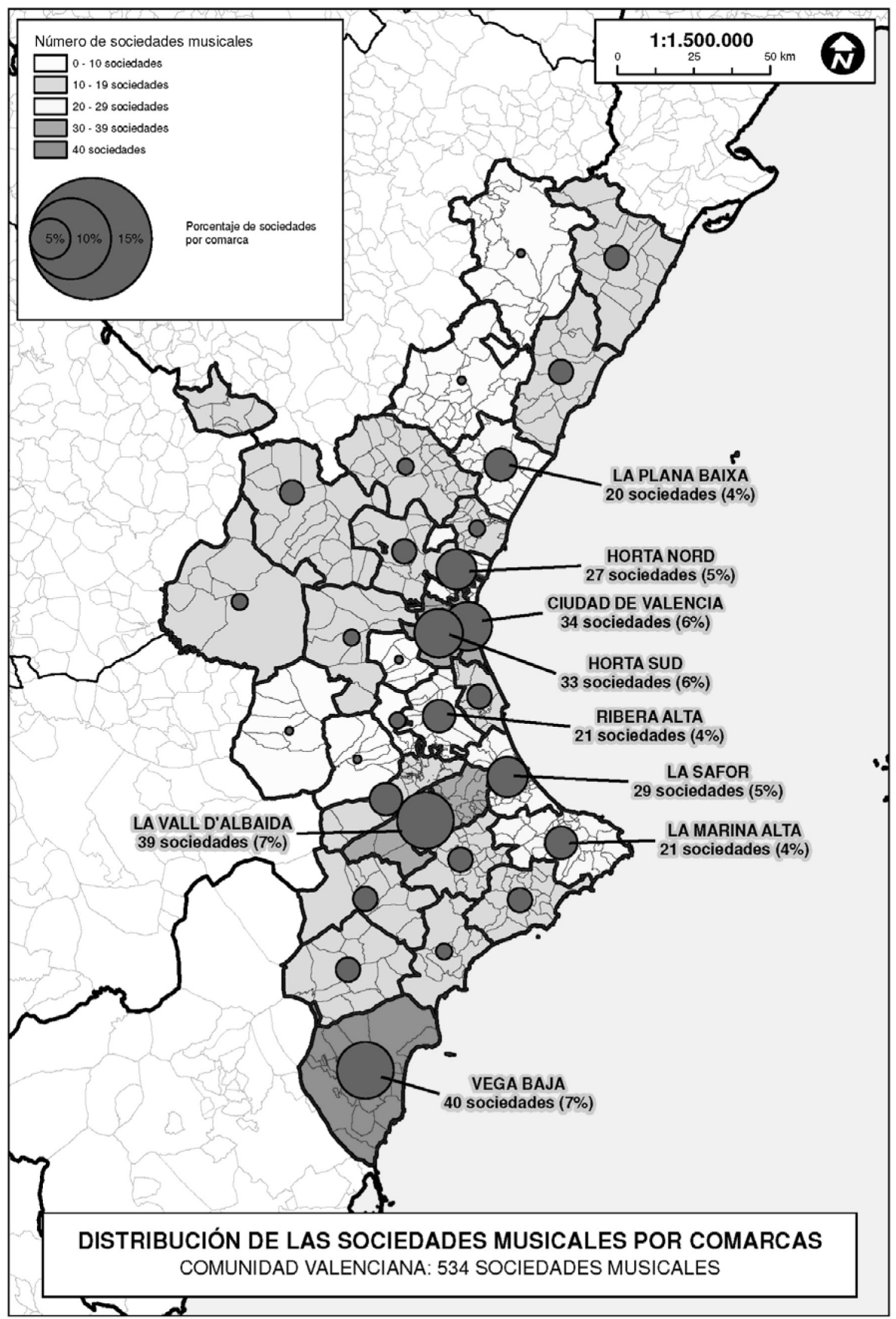

Figura 5. Distribución de sociedades musicales por comarca.

Fuente: elaboración propia. 
crecimiento en número de sociedades de toda la Comunidad Valenciana fue de un 20'29\% en los catorce años anteriores (Rausell Köster, 2011: 9). Los datos de este año nos dicen que hoy día ninguna de las tres provincias queda cerca de esa marca. En segundo lugar, también se sugiere que el mayor potencial de desarrollo lo tienen Alicante y Castellón, dado que tienen menos sociedades y que, por tanto, son susceptibles de crear mayor número de ellas.

\section{IMPACTO SOCIAL}

Según hemos visto en los gráficos, mapas y los datos mostrados, la presencia de las bandas en los municipios es un fenómeno realmente relevante y que da muestra de la vinculación de la población valenciana en estas agrupaciones musicales. Prueba de ello es que de los 542 municipios de la comunidad valenciana, 534 de ellos tienen bandas, lo que supone que un 98 '5 \% de los municipios las tienen. En términos de población vinculada es también un hecho importante ya que el número de músicos en cada una de ellas no es pequeño, no son bandas de pocos músicos, al contrario.

Este fenómeno da a entender que existe una fuerte vinculación de muchas personas de la población dedicada a la banda, aportando conocimiento, trabajo y experiencia (el "saber hacer"). Las estadísticas pertenecientes a este año 2011 recogen que existen 40.000 músicos federados y en torno a 150.000 personas vinculadas.

Lo dicho anteriormente implica que el fenómeno de las bandas es fundamentalmente asociativo, es decir, requiere una unión social espontánea y organizada alrededor de la música. Ello contribuye a crear una notable cohesión social en torno al fenómeno musical que, más allá de las fiestas y celebraciones anuales, une a la comunidad. A su vez, esto significa que existe una demanda social de música que, sin embargo, ha crecido sin ayuda de la acción pública por haber quedado al margen de las políticas culturales recientes, lo cual le dota de un mayor valor si cabe (Rausell Köster, 2011). De esta manera, las bandas musicales se convierten en elementos vertebradores de la comunidad $\mathrm{y}$, por tanto, en agentes de contacto entre sus diferentes estratos. Esto es especialmente valioso cuando se trata de la población joven, por lo general más inclinada a otras expresiones culturales más pasivas. Además, puesto que el acceso a este tipo de música carece de restricciones, ya sea a su ejecución o a su aprendizaje, podemos decir también que se trata de un auténtico fenómeno cultural democrático.

4. LA RECUPERACIÓN ECONÓMICA DE LAS SOCIEDADES MUSICALES DE LA COMUNIDAD VALENCIANA

La cultura, como sector económico, forma parte de los sistemas económicos territoriales porque la creación, producción, distribución y consumo de cul- 
tura genera riqueza, también supone un vínculo directo con el sector turístico y este sector tiene un profundo desarrollo en la comunidad valenciana. Las actividades culturales musicales pueden contribuir a la economía local en varios sentidos; lo primero porque estas actividades tienen un valor añadido ya que generan ocupación y porque arrastran a otras pequeñas empresas de proveedores: tiendas de compra y reparación de instrumentos, tiendas de artículos y partituras, viajes, guías, etc. La cultura o la práctica cultural de los ciudadanos es una parte importante en la composición de los niveles de calidad de vida colectiva, el consumo cultural da respuesta a ciertas demandas de identidad. En el caso que nos ocupa consumir música de banda para los propios ciudadanos es materializar su propia sensibilidad, su capacidad creativa y comunicativa.

Las Sociedades Musicales no persiguen ninguna finalidad de lucro; de hecho están en déficit permanente. Sin embargo, al margen de los efectos de las bandas como dinamizador sociocultural tienen también efectos económicos de arrastre muy relevantes. En este sentido, las Sociedades Musicales constituyen sin lugar a dudas el fenómeno asociativo más extendido y significativo de la Comunidad Valenciana, tanto desde un punto social como económico. El resultado más visible de estas asociaciones musicales son los conciertos. Veamos los diferentes aspectos de su repercusión económica.

Los presupuestos de las sociedades musicales federadas sumaban en 2009, según el último estudio realizado por la Universidad de Valencia era de 46.606.518€ como ingresos y se reflejaban 45.284.899€ de gastos. La distribución de estos presupuestos por provincias de la comunidad autónoma era de un $65,4 \%$ para la provincia de Valencia, un $12,6 \%$ para Castellón y un $22 \%$ para Alicante. Los ingresos se generan en un $77 \%$ a cambio de prestación de servicios y el $21 \%$ provienen de subvenciones públicas, por lo que estos datos justifican la idea de que generan ingresos de manera muy significativa y no dependen exclusivamente de aportaciones externas. Las aportaciones públicas proceden de la Generalitat Valenciana, los ayuntamientos y las diputaciones. Las cifras oscilan entre 9,5 y 10 millones de euros. El resto de ingresos provienen de las matrículas de las escuelas de música, las actuaciones y convenios de los ayuntamientos, las cuotas de los socios, las loterías y rifas, alquiler de sus propios espacios, etc.

La provincia de Valencia tiene asignado el mayor porcentaje del presupuesto para toda la comunidad autónoma, el más importante con diferencia y en relación a las otras dos provincias de Alicante y Castellón. Valencia tiene en su provincia la mayor cantidad de sociedades musicales con un $60 \%$ (316 bandas), mientras que Alicante cuenta con un 26\% (136 bandas) y Castellón con un 12,60\% (77 bandas) (Fuente: Econcult). 


\section{EMPLEO Y OCUPACIÓN}

El estudio económico que se ha realizado para las sociedades musicales y que hemos citado anteriormente, muestra una serie de datos muy reveladores relacionados con el empleo real, potencial y voluntario. Se han recogido una cantidad nada despreciable de 1.840 empleos directos, 573 empleos inducidos y 162 empleos indirectos, el trabajo voluntario es de 380 puestos de trabajo a tiempo completo. Estas cifras nos dicen que son entes que sí emplean personas, que están en permanente crecimiento y que es un fenómeno tan arraigado que asegura siempre la renovación de estos empleos y funciones a desempeñar por cualquier socio o músico vinculado con las actividades de la sociedad musical. Estos trabajos están relacionados con la administración y gerencia, con los profesores de las escuelas musicales, los directores de las bandas y otros trabajos auxiliares. Lamentablemente sólo el 1\% de este empleo se dedica a la gestión cultural por parte de gestores profesionales.

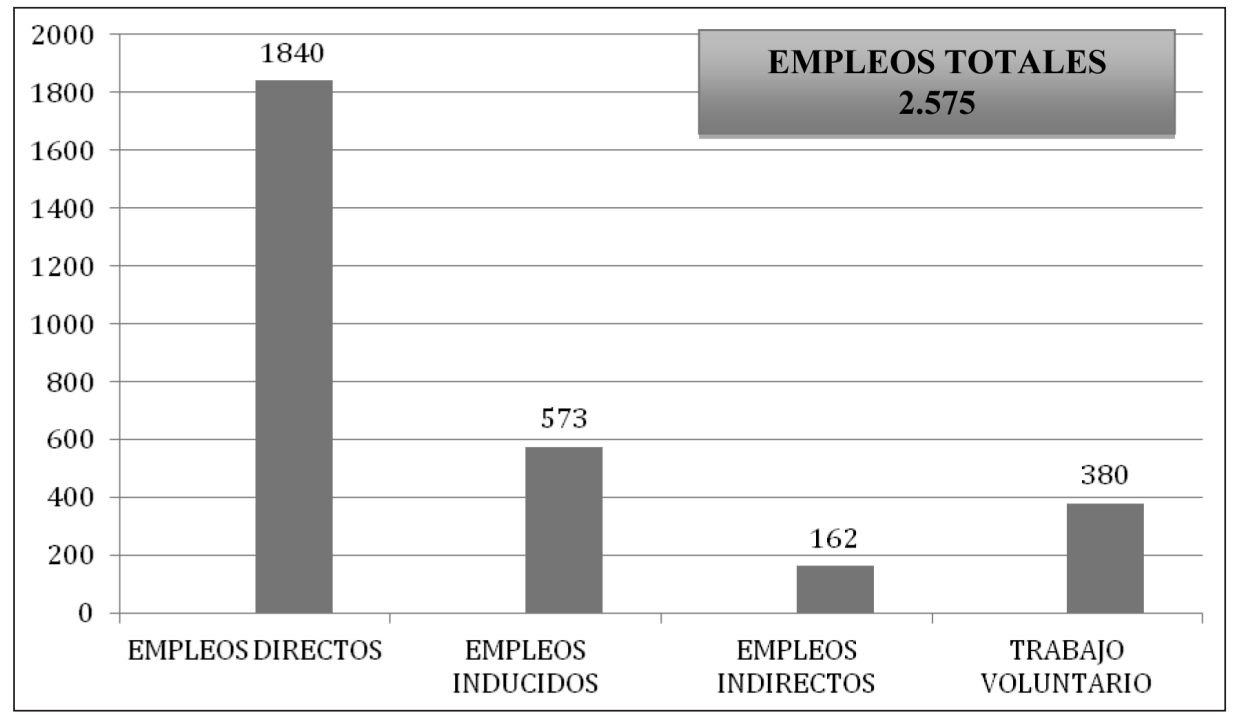

Figura 6: Empleos generados en las sociedades musicales.

Fuente: elaboración propia.

\section{LA MÚSICA COMO HERRAMIENTA DE DESARROLLO LOCAL}

La naturaleza actual de las Sociedades Musicales se enmarca en el sector de la economía no lucrativo; sin embargo, tienen una gran relevancia social y económica.

La cultura es un sector estratégico de futuro. La creación cultural, y en nuestro caso la musical, así como las personas a ellas vinculadas y los valores 
intangibles que llevan consigo, están cada vez más inmersas en la sociedad. La importancia de la cultura en la transformación social de los territorios le infunde una plusvalía social que tiene, a su vez, una repercusión económica. La cultura es un sector clave dentro del proyecto social de un territorio, junto con el bienestar social, la cooperación, los deportes, la educación y la sanidad, contribuyendo al desarrollo y al dinamismo, también a la creatividad, y crea una red rica de relaciones sociales.

Es necesario invertir en una planificación estratégica para así poder dar continuidad al patrimonio autóctono. Es igualmente crucial saber adaptarse a los cambios sociales y tecnológicos para preservar a largo plazo el patrimonio cultural. En este proceso, son necesarias nuevas formas de pensamiento: se necesita un proceso creativo y una aportación de ideas que puedan generar decisiones tácticas sustanciales y efectivas. No hay que frenar las posibilidades de crecimiento económico y desarrollo social. Dentro del campo de la cultura en general, la música es susceptible de ser ofertada dentro de programas de promoción turística. Podemos ofrecer experiencias basadas en el patrimonio, que es la música misma, y convertirlo así en un potencial para la comunidad local. La administración debe mantener este patrimonio como una obligación de mantener la cultura propia, fomentarla y ponerla en valor; pero debe existir un modelo de gestión público-privado para que estos recursos que se reciben de la administración se canalicen y se potencien y ayuden a iniciativas privadas a mejorar los ingresos. La calidad y la imagen de la música pueden convertirse en un reclamo, en una marca de calidad. Las bandas de música son un patrimonio cultural que está en un proceso de transformación de ideas y estructuras. Las políticas culturales deben encauzar las actuaciones sobre estas actividades culturales musicales desde el ámbito local.

El motivo que justifica este enfoque es que en el ámbito local es donde el ciudadano se desarrolla y donde se producen la mayor cantidad de demanda de conciertos y servicios educativos que ofrecen estas organizaciones musicales. Su territorio propio -ya se trate del pueblo, la ciudad o la comarca- es donde se desarrollan las políticas culturales y donde estos ciudadanos pueden participar como miembro de esta sociedad. Sin embargo, debería entenderse que el ciudadano es un cliente para que las bandas y las sociedades musicales generen nuevas actividades y que reciban la aprobación, gusto y sean consumidas por los ciudadanos como productos musicales de calidad. Resulta necesario detectar públicos potenciales y que estas bandas, a su vez, puedan estar bien gestionadas económicamente y generar beneficios para que pudieran invertirse en mejoras de todo tipo: inversión en las escuelas musicales, instrumentales, mejora de producción de conciertos, infraestructuras, etc. Por tanto, es necesario cobrar por estos conciertos que las bandas ofrecen, ya que la programación y mantenimiento no puede ser siempre sostenida únicamente por las administraciones. Para ello, proponemos que las Sociedades Musicales se administren 
como empresas, dado que su actual estado administrativo y jurídico determina sus características, funciones y actividades. Tanto la Agenda 21 de la Cultura como el marco legislativo del Instituto Valenciano de la Música deben desarrollar principios e iniciativas de protección y desarrollo de la música valenciana. Así, el sistema cultural local ha de servir como herramienta de diagnóstico e intervención, tal y como se ha propuesto en varios estudios sobre cultura y desarrollo (Rausell Köster, 2007).

BIBLIOGRAFÍA

CASARES RODICIO, E. (coord.) (2006): Diccionario de la música valencia$n a, 2$ vols. Valencia, Fundación Autor.

RAUSELL KÖSTER, P. (comp.) (2007): Cultura. Estrategia para el desarrollo local. Madrid, Agencia Española de Cooperación Internacional para el Desarrollo, Ministerio de Asuntos Exteriores.

RAUSELL KÖSTER, P. (dir.) (2011): Estructura presupuestaria, dimensión económica e impacto económico de las sociedades musicales en la Comunidad Valenciana. Valencia Universidad de Valencia.

RUIZ, V. (1993): Historia de las Sociedades Musicales de la Comunidad Valenciana: las bandas de música y su federación. Valencia, Federación de Sociedades Musicales de la Comunidad Valenciana.

UNESCO (2003): Convención para la salvaguardia del patrimonio cultural inmaterial. http://portal.unesco.org/culture.

VV.AA. (2002): II Congreso General de Sociedades Musicales de la Comunidad Valenciana. Valencia, Federación de Sociedades Musicales de la Comunidad Valenciana.

VV.AA. (2006): Agenda 21 cultura. Un instrumento para el desarrollo: principios, metodologías y estrategias para su implementación en el territorio. Castellón, Universitat Jaume I. 